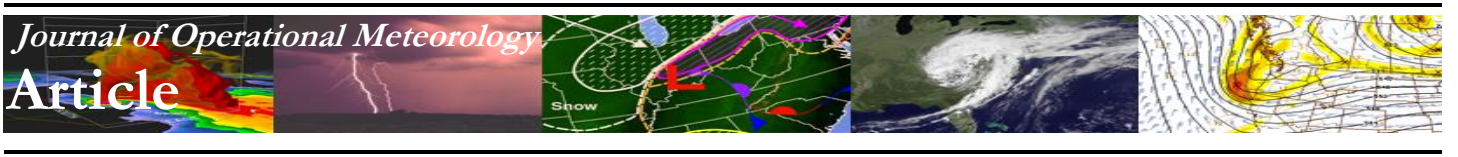

\title{
A Parameter Weighting Scheme for an Automated Weather Effects Decision Aid
}

\author{
RICHARD J. SZYMBER, TERRY C. JAMESON, and DAVID I. KNAPP \\ United States Army Research Laboratory, White Sands Missile Range, New Mexico
}

(Manuscript received 31 December 2012; in final form 3 April 2013)

\begin{abstract}
For the past twenty years Army field intelligence analysts and staff weather officers assigned to combat weather teams have utilized a decision support tool called the Integrated Weather Effects Decision Aid (IWEDA). The IWEDA system ingests weather forecast model grids, applies a rules/thresholds database to the grids, and produces color-coded overlays for terrain map backgrounds. These map overlays quickly indicate the severity of the weather impacts on Army weapon and support systems, and have provided a valuable tool for mission commanders to plan battlefield operations.

Although it is a useful tool, the basic IWEDA concept has not been updated since its inception, and its capabilities have fallen behind the vastly improved numerical weather prediction models and computing platforms that are available today. Its coarse color-coded map indicators are a simplistic green/amber/red scheme, all weather parameters are treated with equal weight, and there is no means of accounting for how many model parameters are contributing to the adverse weather effects. The current research describes a new composite scoring system by which weather parameters can be assigned different weights; an accounting is made for the number of parameters contributing to the adverse weather, and much greater color granularity can be applied to the IWEDA map overlays. The higher color granularity and adjustable parameter weights are expected to afford much greater flexibility to commanders and intelligence analysts as weather effects are incorporated into planning battlefield operations. The new capability also is suitable for use in comparable operational civilian weather impacts technologies.
\end{abstract}

\section{Introduction}

The Integrated Weather Effects Decision Aid (IWEDA), originally developed by the United States Army Research Laboratory (ARL) in 1992, has been fielded on an intelligence and electronic warfare system called the Integrated Meteorological System (IMETS) since 1997 to provide tactical weather support to the United States Army, as described in Szymber (1997). Sauter et al. (1999) have documented the capabilities of the IWEDA software and Raby et al. (2003) have summarized its verification/validation studies. The Army IWEDA rules database and model software developed by the ARL officially were certified and accredited for operational Army use by the United States Army and Intelligence Center and Fort Huachuca (Department of the Army 2006). Underlying information on the IWEDA assumptions, criteria, development, description, and rules is contained in Szymber (1998, 2008).
User feedback over the years of IWEDA use has identified the needs for potential improvements including a need to (1) derive an overall mission impact due to adverse weather conditions, rather than simply presenting the worst-case conditions and (2) better represent the discrete color-coded impact values (IVs). The existing IWEDA rules color-code for the IVs has always employed three discrete colors, as depicted in Fig. 1 and defined here (Department of the Army 1992): (1) green for favorable or low/negligible impacts (weather impact system degradation less than $30 \%$ ), (2) amber for marginal or moderate impacts (weather impact system degradation equal to 30-70\%), and (3) red for unfavorable or severe impacts (weather impact system degradation greater than $70 \%)$.

A parameter weighting scheme (PWS) has been proposed to address the need for IWEDA to provide more quantified information on the magnitude of

Corresponding author address: Mr. Terry C. Jameson, United States Army Research Laboratory, RDRL-CIE, White Sands Missile Range, NM 88002-5501

E-mail: terry.c.jameson.civ@ mail.mil 


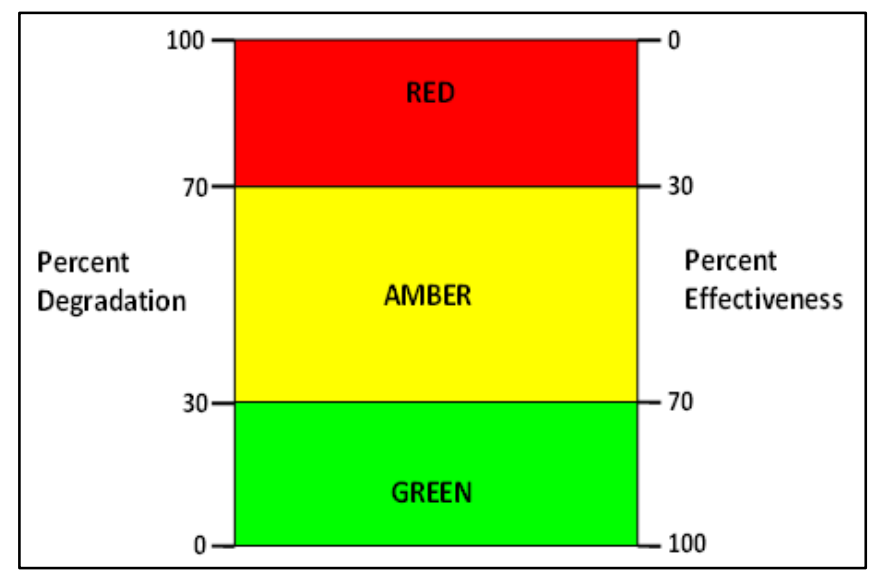

Figure 1. The current IWEDA "stoplight" color-code scheme, with corresponding percent of system degradation due to adverse weather (left) and the percent system effectiveness (right). Click image for an external version; this applies to all figures hereafter.

weather impacts. The novel PWS has been prototyped (as a Java code) based on an overall/total mission impact score in each forecast model grid cell [called the cell impact score (CIS)]. The CIS is derived using the original IVs output by IWEDA and then applying user-input weights assigned to the individual weather parameters. It ranges in value from $0-1.0$, indicating a spectrum of weather impacts ranging from no degradation up to complete degradation. The PWS outputs a continuum of ten discrete colors that corresponds to the spread of CIS values (three minimal impact colors, four moderate impact colors, and three severe impact colors; see Fig. 2). Relative to the previous system, the CIS is a more highly granular measure or continuum of the weather impact; thus, the PWS approach yields substantially more information/detail compared to the current three-color IWEDA system. It will allow the user to prioritize the impacts by assigning greater or lesser significance to certain weather parameters based on tactical mission considerations (i.e., the tactical scenario and mission profile), the near-term forecast weather conditions, evolving synoptic-scale patterns, and local weather effects and terrain influences. The ultimate objective of the PWS is to give the decision maker an improved overall assessment of weather impacts on his/her plans - while highlighting the potential worst case conditions - based on prioritizing the importance of specific weather parameters on the mission.

This paper describes the following: (1) more detail about the weather impacts, as well as the limitations of the current IWEDA methodology; (2) specifics about how the proposed PWS is formulated; (3) the construct of a case-study comparison between IWEDA and the
PWS; (4) the results of the case-study comparison; and (5) conclusions and ongoing work to enhance the PWS concept.

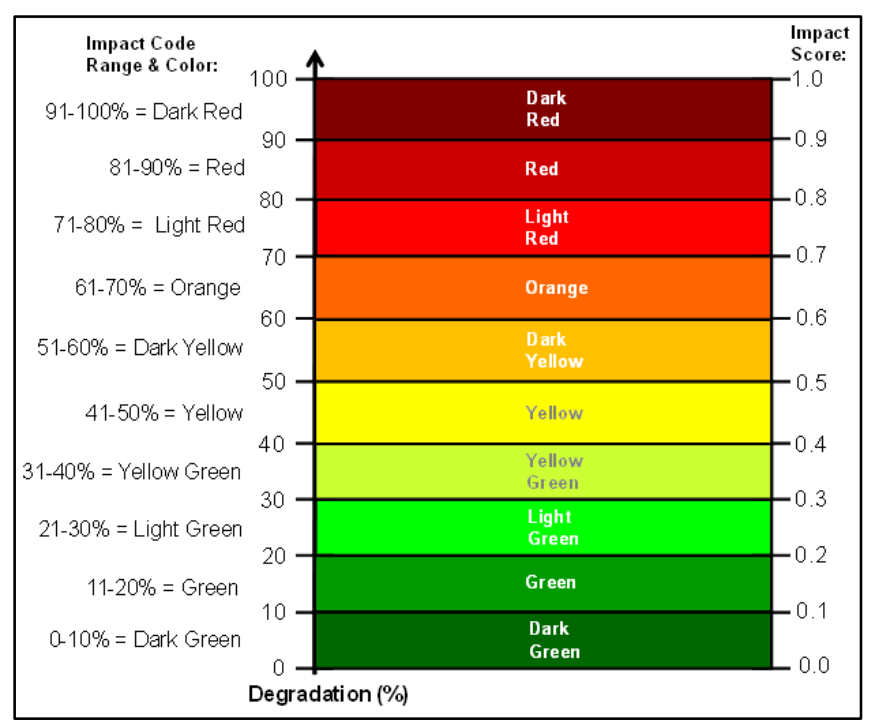

Figure 2. High-granularity PWS impact color code. The CIS scale is indicated on the right-hand vertical axis and the corresponding percent mission degradation due to adverse weather is depicted on the left-hand vertical axis.

\section{The weather impacts process}

To better understand how the proposed PWS works, as well as the advantages it may afford over IWEDA, it is useful to have a somewhat in-depth description of how weather impacts are derived. When IWEDA is invoked in IMETS, gridded numerical weather prediction (NWP) model forecast data in hourly and/or three-hourly increments are obtained via satellite, including model basic parameters (e.g., temperature, moisture, wind components, etc.), and post-processed, derived parameters (e.g., wind direction/speed, relative humidity, visibility, etc.). These forecast data are used to build a fourdimensional gridded meteorological database, which is applied in IWEDA. Then, a system or set of systems is selected. For example, a set of two different types of helicopters, a fixed-wing jet, or associated ground support vehicles might be selected for an air assault mission. In each model grid, the forecast parameters are then compared against threshold values from the IWEDA rules database. If a forecast value exceeds a "marginal" or "unfavorable" rules threshold, a 1 or a 2 IV is generated, corresponding to an amber or red color-coding in that grid cell on the map, respectively (see Fig. 3). For example, if the forecast surface wind speed in a particular grid cell is $16 \mathrm{~m} \mathrm{~s}^{-1}$, and the 


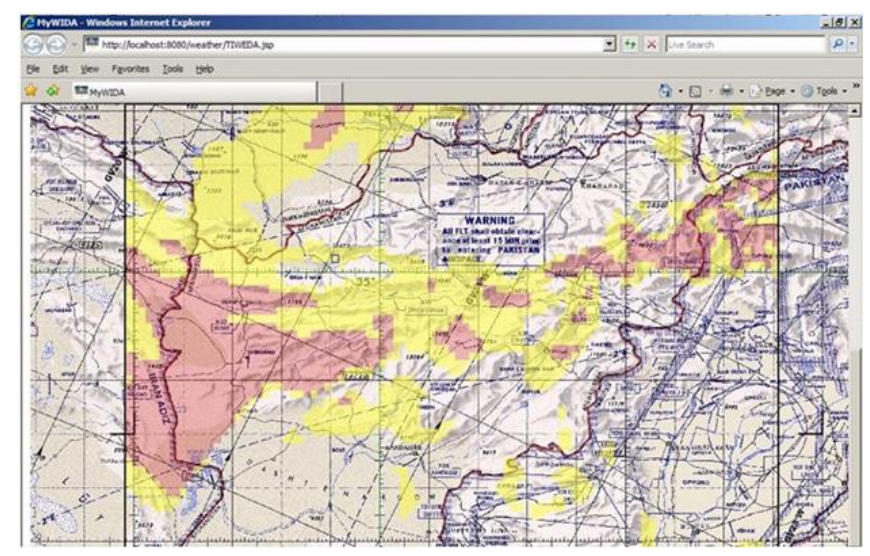

Figure 3. Example of IMETS/IWEDA weather impacts display.

unfavorable impact threshold value for a helicopter is $15 \mathrm{~m} \mathrm{~s}^{-1}$ (this is not an actual IWEDA database threshold, but an example only), the forecast exceeds the threshold and a red color-coding is depicted in that cell. For each model grid point, all the cells in the vertical above that point are interrogated if associated with any upper-air parameters, and the worst case impact is assigned to that grid point cell (i.e., summing all the impacts in the vertical). Additionally, all the cells in the horizontal are interrogated, and the worst case impact occurring is assigned that corresponding color in the weather effects matrix (WEM) chart (i.e., summing all the impacts in the horizontal). Thus, the impacts overlay is a display of the spatial distribution of impacts over the area of interest (AOI) for a specific forecast period, and the WEM displays a time series of the worst case impact in the AOI spanning all forecast periods.

There are three primary limitations to this process that the PWS is designed to address. The first is that having only three discrete weather impacts is too coarse to yield the most useful information. For example, a red depiction in a grid cell indicates only that the $70 \%$ degradation threshold has been exceeded, when the actual degradation could be anywhere from 70 to $100 \%$. Second, all threshold parameters in IWEDA are treated with equal weight, when in reality some parameters could be of much more concern to the mission planners than others, and/or could have more or less overall influence on the weapons system or tactical operation. For example, high surface wind speeds could be much more of an issue than low visibility. Last, a marginal or unfavorable weather impact will be depicted regardless of how many thresholds were exceeded. Intuitively, multiple thresholds being exceeded would have a more adverse impact on the mission than only one.

\section{Construct of the PWS}

\section{a. Mission-oriented versus system-oriented approach}

The current IWEDA is structured along a systemsoriented hierarchy. To set up the weather effects analysis, a system or set of systems must be selected (e.g., the individual air and ground vehicles for the air assault mission described earlier). Then, weather parameter thresholds that apply to each system (as well as to all its sub-systems, and in some cases even down to the sub-system component level) are compared against applicable forecast grids. This is a somewhat cumbersome approach, often involving numerous IWEDA rules/thresholds. The PWS is instead structured along a mission-oriented hierarchy. A concise set of weather parameters is designated that applies to an overall mission, for example surface wind speed, surface temperature, visibility, and cloud ceiling might be applied to the air assault mission.

\section{b. Mission-set weather parameters and threshold values}

Szymber (2008) cites the official Army weather data support requirements and critical threshold values and impacts as defined and validated by Army user agencies. This information was used to develop a special set of Army mission command IWEDA rules. The Army mission command rules set is based on essential weather/environmental elements and critical thresholds impacting major Army battlefield systems, operations, and tactics. By definition, essential data (weather/environmental parameters and thresholds) can affect user systems and operations so significantly that a user reaction is required when critical values of these elements are exceeded. From the 16 Army mission areas, 12 general categories of essential weather and environmental parameters were identified (Table 1). Threshold values for this mission set of parameters were designated that pertain to the lowest allowable threshold. To clarify this point, assume two different helicopters are assigned to a mission. If one helicopter can tolerate a surface wind speed of $13 \mathrm{~m} \mathrm{~s}^{-1}$ before conditions are unfavorable (red) for takeoff, and the other aircraft involved in the mission can tolerate $15 \mathrm{~m} \mathrm{~s}^{-1}$, the threshold is set at the lower value. As can be seen from Table 1, a particular category might consist of a single parameter (such as visibility), or of multiple parameters (such as clouds, which is comprised of both cloud ceiling and sky cover). It is recognized that not all of these weather parameters are 
Table 1. Significant weather parameters affecting the 16 Army mission areas.

\begin{tabular}{|c|c|}
\hline Parameter & Attribute(s) \\
\hline Clouds & Cloud ceiling and sky cover \\
\hline Relative humidity & Surface \\
\hline Icing & Aloft \\
\hline Illumination & Ambient \\
\hline $\begin{array}{c}\text { Obstructions to } \\
\text { vision }\end{array}$ & Fog, blowing dust/sand/snow \\
\hline Precipitation & Rain, snow (and snow depth), freezing rain, hail \\
\hline Severe weather & Wind, hail, flooding, tornadoes, hurricanes \\
\hline Air temperature & Surface \\
\hline Thunderstorms & Distance from site; probability of lightning \\
\hline Turbulence & Aloft \\
\hline Visibility & Surface \\
\hline Wind & Speed and direction; surface and aloft \\
\hline
\end{tabular}

output directly by current NWP models, nor can they be derived by existing post-processing algorithms. For example, although cloud ceiling may be a model postprocessed value, the amount of sky cover may not be. Table 1 simply represents a comprehensive listing of all weather parameters that can have a significant impact on Army operations, spread across the 16 mission areas.

\section{c. The parameter weighting concept}

Army mission planners must be aware of the weather factors that will affect their operations, ensuring the greatest chance of mission success. Use of the PWS involves determining which forecast weather parameters might have the greatest impact on assigned missions, and, therefore, should be given extra consideration and special weighting in operational planning. Parameter weighting is desirable when operators and planners have a priori knowledge of significant weather events or factors that will impact their AOI and conduct of operations. Factors influencing the importance of weather parameter selection and weights involve the following: (1) the weather parameter's criticality to the tactical situation and mission profile (especially with respect to time and location); and (2) the parameter's importance from the standpoint of the weather situation/scenario, based on the synoptic forecast and long-range outlook, climatology and season, geography, and local terrain influences.

A basic premise of the PWS is that the user determines which parameters to weight. The parameters to be weighted are selected out of the full set of associated rules-based weather parameters for a selected mission area. For each of the parameters that will be weighted, the operator assigns an appropriate weighting category, choosing from three options: lightly weighted, moderately weighted, or heavily weighted. Multiplication factors are assigned accordingly by the PWS that are referenced to the equal-weight amount. Based on the number of parameters chosen for weighting, the program automatically calculates the equal parameter weight, then the light, moderate, and heavy parameter weights. Any remaining weather parameters that are not intended for the analysis are zero-weighted and have no effect in the PWS calculations.

\section{d. Parameter weights development}

The sum of the weights for those parameters included in the analysis (the non-zero-weighted parameters or num_non_zero_wgtd_params) must equal 1.0. If all parameters were to be weighted equally, that equal weight (eq_param_wgt) is found by dividing 1.0 by the number of parameters. As coded in the PWS Java program this is:

eq_param_wgt $=\frac{1.0}{\text { num_non_zero_wgtd_params }}$

This equal parameter weight is a variable value, being a function of the number of parameters included in the particular analysis.

For ease of selection the parameter weights have been separated into three categories that are referenced to the equal parameter weight: light, moderate, and heavy (per section 3c). The first two weights are found by applying a multiplication factor to the equal parameter weight. For the sake of convenience and simplicity in the PWS conceptual development, the moderate parameter weight (mod_wgt) was chosen simply to be the same as the equal weight, that is its multiplication factor is 1.0 (which could be adjusted somewhat pending further research):

$m o d \_w g t=e q \_p a r a m \_w g t \times 1.0$

To further facilitate the initial PWS investigations, the light parameter weight (lgt_wgt) was somewhat arbitrarily selected to be one-half of the equal weight:

$l g t \_w g t=e q \_p a r a m \_w g t \times 0.5$

With the various parameters falling into one of the three weighting categories, the sum of their weights still has to equal 1.0. More specifically, the number of lightly weighted parameters (num_lgt) multiplied by 


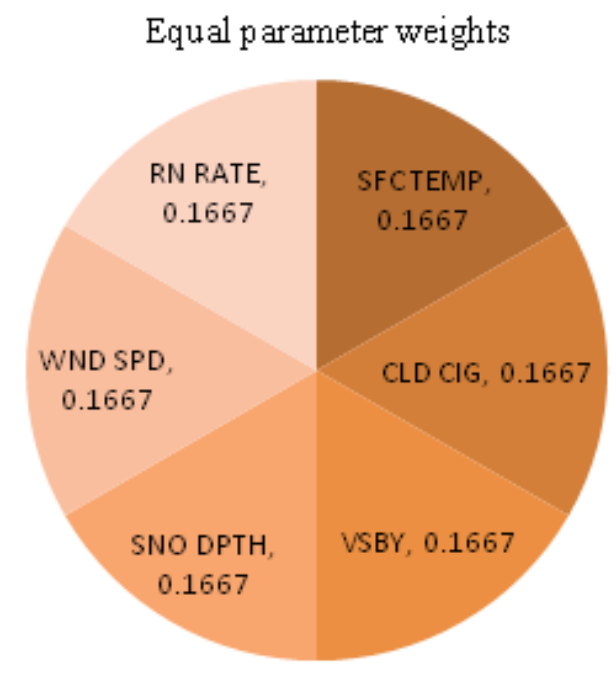

Total number of parameters equals 6 Lightly weighted parameters equals 3 Moderately weighted parameters equals 1 Heavily weighted parameters equals 2

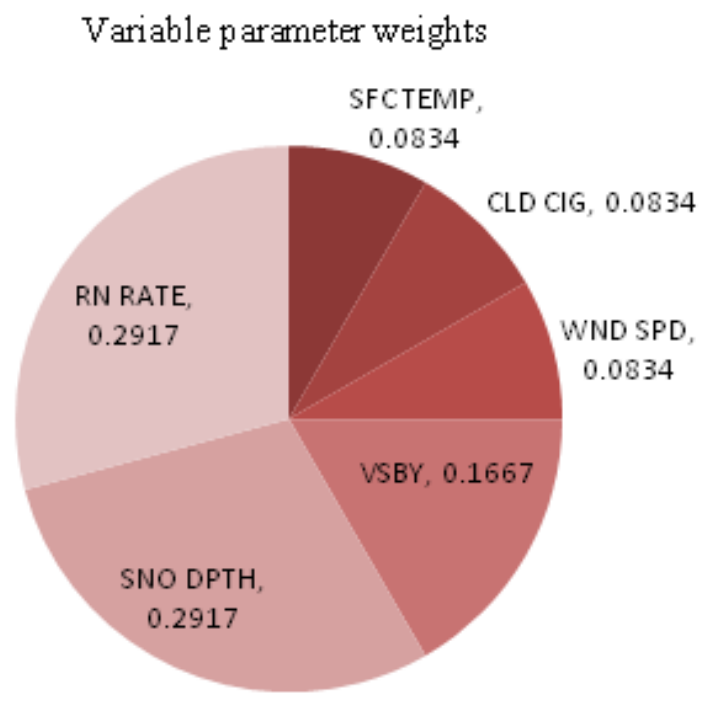

Figure 4. Pie-chart depictions of the division of parameter weights (top $=$ equal parameter weights; bottom $=$ variable parameter weights).

the light-weighting factor, plus the number of moderately weighted parameters (num_mod) multiplied by the moderate-weighting factor, plus the number of heavily weighted parameters (num_hvy) multiplied by the heavy weighting factor (hvy_wgt) must equal 1.0, as follows:

$$
\begin{gathered}
t o t \_w g t=\left(n u m \_l g t \times l g t \_w g t\right)+\left(n u m \_m o d \times\right. \\
\left.m o d \_w g t\right)+\left(n u m \_h v y \times h v y \_w g t\right)=1.0
\end{gathered}
$$

In the development of the three weighting factors, the remaining unknown is hvy_wgt. Equation (4) can be rearranged to solve for $h v y_{-} w g t$ by the use of (2) and (3), which yields the following:

$h v y \_w g t=\frac{1.0-e q \_p a r a m \_w g t\left[0.5 \times n u m \_l g t+n u m \_m o d\right]}{n u m \_h v y}$

A pie chart depiction of how the parameter weights are determined using the above equations is shown in Fig. 4. In this particular example, six parameters were chosen to be weighted in the analysis - three of which were to be lightly weighted, one of which was to be moderately weighted, and the remaining two being heavily weighted. Any remaining parameters from the rules set that are not selected for inclusion in the analysis are zero-weighted, and thus have no impact upon the final CIS.

\section{e. Derivation of the CIS}

The CIS is actually the normalized sum of the weighted IVs. The meaning of this phrase will be broken down in the following paragraphs.

Within the prototype Java PWS code, IV arrays similar to those produced by IWEDA are read for multiple layers (simulating an array from each forecast model level). In other words, a three-dimensional array of $0 \mathrm{~s}, 1 \mathrm{~s}$, and $2 \mathrm{~s}$, for each parameter being weighted, is input into the PWS. For example, if six parameters are being included in the analysis, six 3-dimensional IV arrays are input.

For each cell and each parameter, the IV is multiplied by its parameter weight. These products are called the weighted impact values (WIV). The WIVs for all parameters are then summed, obtaining the sum of the weighted impact values (SWIV). The SWIV is found for each individual cell.

Finally, the SWIV for each cell is divided by 2.0, the quotient being the CIS. Dividing by 2.0 normalizes the CIS because 2.0 is the maximum value possible for the SWIV. This would occur only if the IV for every parameter (for that cell) was 2.0. In that case the computed CIS would be 1.0 (its maximum value). An example CIS computation for a grid cell is shown in Table 2. 
Table 2. Example CIS computation for six weighted parameters that results in a CIS of 0.60 for this grid cell. Impact values (IVs) correspond to negligible (0, green), marginal (1, amber), and unfavorable (2, red).

\begin{tabular}{|c|c|}
\hline Variable & Value/Weight \\
\hline Equal parameter weight & 0.1667 \\
\hline Moderate parameter weight & 0.1667 \\
\hline Light parameter weight & 0.0833 \\
\hline Heavy parameter weight & 0.2917 \\
\hline Surface wind speed weight & 0.0833 \\
\hline Surface temperature weight & 0.0833 \\
\hline Cloud ceiling weight & 0.0833 \\
\hline Visibility weight & 0.1667 \\
\hline Snow depth weight & 0.2917 \\
\hline Rainfall rate weight & 0.2917 \\
\hline Surface wind speed IV & 1 \\
\hline Surface temperature IV & 1 \\
\hline Cloud ceiling IV & 0 \\
\hline Visibility IV & 1 \\
\hline Snow depth IV & 2 \\
\hline Rainfall rate IV & 1 \\
\hline Surface wind speed weighted IV & $1 \times 0.0833=0.0833$ \\
\hline Surface temperature weighted IV & $1 \times 0.0833=0.0833$ \\
\hline Cloud ceiling weighted IV & $0 \times 0.0833=0$ \\
\hline Visibility weighted IV & $1 \times 0.1667=0.1667$ \\
\hline Snow depth weighted IV & $2 \times 0.2917=0.5834$ \\
\hline Rainfall rate weighted IV & $1 \times 0.2917=0.2917$ \\
\hline Sum of weighted IVs $(\max =2.0)$ & 1.2085 \\
\hline Normalized sum of weighted IVs & $1.2085 / 2.0=0.6043$ \\
\hline
\end{tabular}

\section{PWS versus IWEDA IV comparisons}

\section{a. The IV arrays}

In preparation for comparing IWEDA impacts against PWS cell scores, a generic set of IVs was generated (i.e., actual IWEDA data were not used in these analyses). The purpose for using manufactured IV arrays was twofold: (1) avoiding the use of actual IWEDA data permits a free distribution of the research results in the public domain; and (2) the IV arrays were formulated in such a way as to highlight the differences between the legacy IWEDA and the new PWS/CIS concept. Concerning the latter point, the real atmosphere would probably never produce a set of IVs for multiple weather parameters resembling the test dataset. However, this configuration was intended to most effectively assess the potential of the PWS as a weather forecast decision support tool by increasing its contrast with IWEDA output.

Figure 5 displays the IV arrays for six weather parameters that were input to the PWS Java code. These graphics emulate a $10 \times 10$, single-level NWP model grid. For ease of viewing, a terrain map background was not included in the plots. Surface wind speed, surface temperature, and cloud ceiling have identical IVs throughout the domain and are emulating unfavorable weather impacts across the entire northwestern half, as indicated by the red grid cells (Fig. 5). A band of marginal impacts (amber grid cells) extends diagonally across the domain. Finally, the southeastern corner of the domain simulates a region where favorable conditions (for these parameters) exist and there are negligible impacts (green grid cells). The IV pattern is roughly reversed for visibility, with minimal impacts existing in the northwestern half of the domain and only a small area of unfavorable in the southeastern corner. There are no unfavorable IVs for snow depth and rainfall rate (Fig. 5 ), with roughly an equal split between marginal and negligible impacts.

\section{b. The parameter weights}

The parameter weights in this analysis were set as indicated in Table 2; the first three were lightly weighted, the fourth was moderately weighted, and last two were heavily weighted. The weights were assigned in this manner to make the point that, even if a parameter's IV is marginal or unfavorable, it should not make a significant contribution to the final analysis of weather impacts if it is relatively unimportant to the mission planner.

\section{Discussion}

\section{a. Side-by-side comparisons: variable parameter weights}

The comparison between the IVs as would be generated by IWEDA and the CIS values as computed by the PWS is shown in Fig. 6 (left and right plots, respectively). It is immediately apparent that there are no green grid cells in the IWEDA display (left), and in fact, the majority is red (indicating unfavorable, which is greater than or equal to $70 \%$ system degradation). This result highlights one of the issues in IWEDA being addressed by the PWS development; regardless of whether a single parameter generates an unfavorable IV, or whether thresholds for multiple parameters are reached, the same red grid cell is depicted. The PWS array on the right of Fig. 6 tells a much different (and more informative) story. Here the CIS values are being influenced by the number of parameters that are exceeding a marginal or 


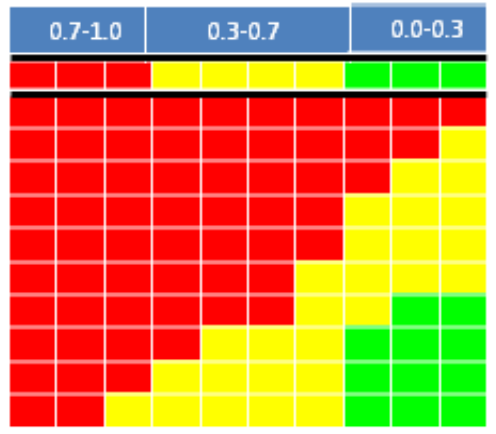

\section{Surface Wind Speed}

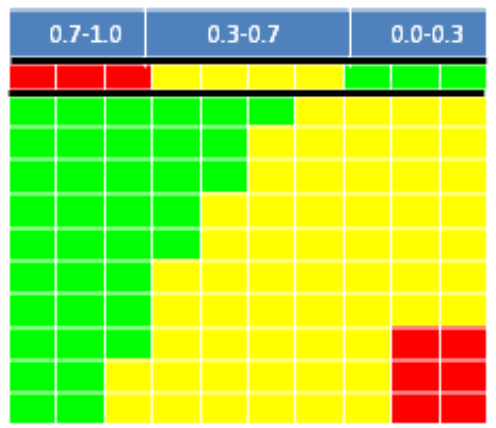

Visibility

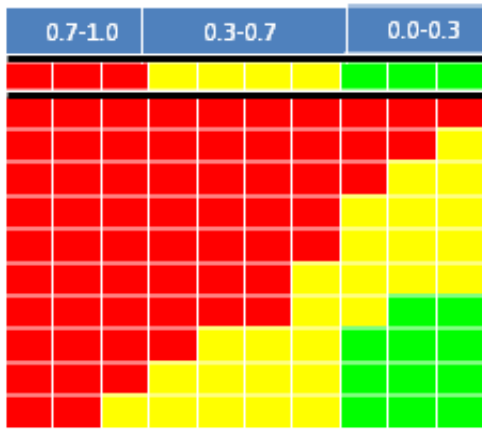

Surface Temperature

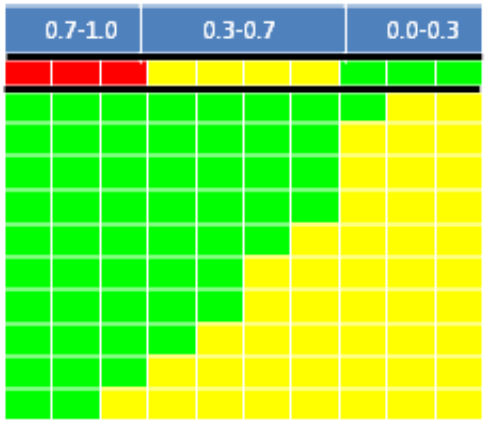

Snow Depth

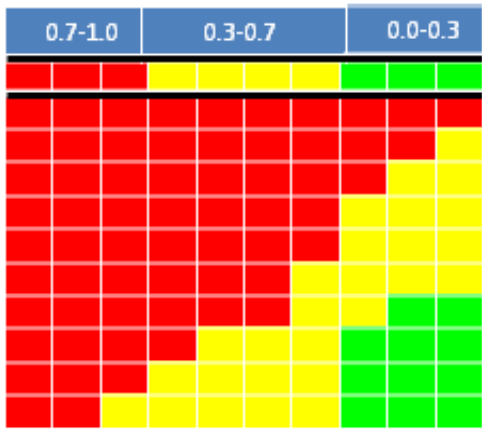

Cloud Ceiling

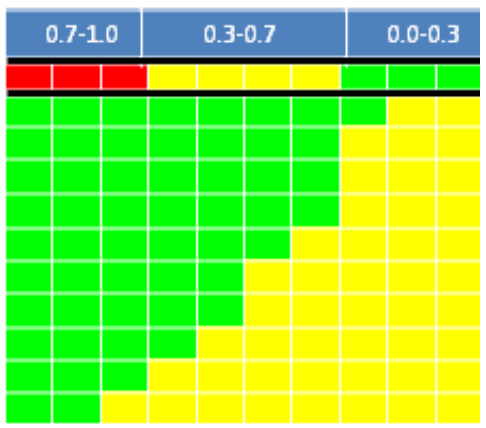

Rainfall Rate

Figure 5. $10 \times 10 \mathrm{IV}$ grid for parameters 1-6 (refer to Table 2).

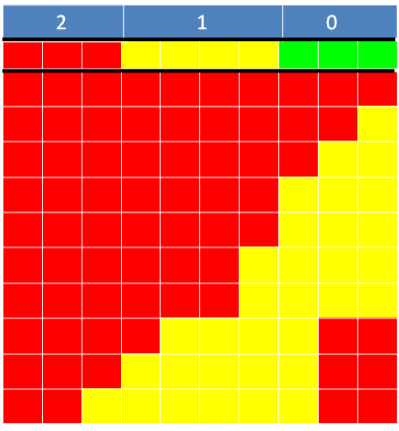

Six-param eter TWEDA analysis

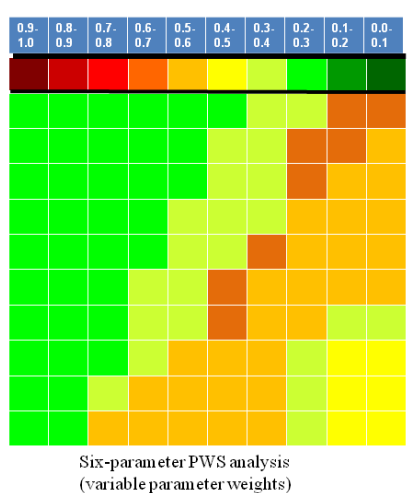

(variable parameter weights)

Figure 6. Side-by-side comparison of legacy IWEDA (left) and prototype PWS output (right). IWEDA and PWS use identical IV arrays as input. Results are from variable parameter weighting.

unfavorable threshold, as well as by how heavily the individual parameters have been weighted. Only a few of the grid cells stretching diagonally through the plot are even in the dark orange range, indicating a mission weather impacts degradation of $60-70 \%$. The yellowgreen and green cells in roughly the northwestern half of the figure depict a mission degradation of $20-40 \%$ as opposed to a $70 \%$ degradation produced by IWEDA in those same cells. This is a more realistic assessment of the overall weather impacts, taking into account how many parameter thresholds were reached in each cell and how important those parameters were to a given mission.

It should be noted that as more parameters are selected to be heavily weighted, equation (5) approaches an equal-weighting configuration. The original intent of the PWS development was to enable analysts and decision makers to emphasize the impact of the most critical weather parameter(s). As a result, the authors suggest that one, or at the most two, MET parameters are heavily weighted in any PWS analysis.

\section{b. Side-by-side comparisons: equal parameter weights}

Having the ability to vary the parameter weights in the CIS computations is a potentially valuable tool for mission planners. However, as was shown in the previous example, it is possible to mask the severity of certain weather impacts simply by lightly weighting those parameters in the PWS setup. Also, in some 

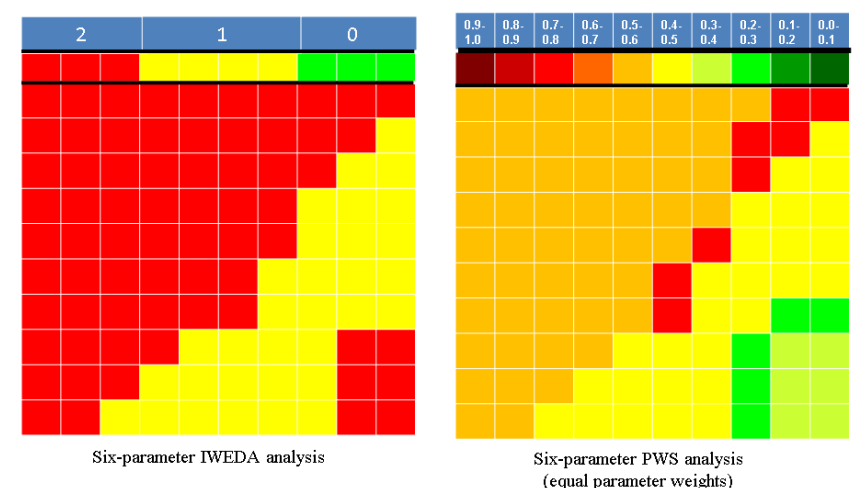

Figure 7. Same as Fig. 6 except results are from equal parameter weighting.

cases the mission planners might not have a priori knowledge that would enable a distinction to be made between the parameter weights. For these reasons, the Java code includes an option of equally weighting all parameters in the analysis. An equal-weighting CIS computation was run using the same IV input as described earlier; the results are shown in Fig. 7. In this case, several of the grid cells remained red, and the northwestern block of cells that before had green shading now has CIS values in the 0.5-0.6 range (yellow-orange). Thus, by equally weighting the parameters, most of the grid cells that were depicted as unfavorable by IWEDA experienced an increase in the CIS values, indicating more severe weather impacts.

\section{Conclusions}

This report introduces a new concept for depicting more granularity in weather impacts information than standard stoplight color-coded decision support tools. The use of a new PWS is applied to the IWEDA decision support tool IV output, whereby various key weather parameters are weighted to define more or less mission impact significance than others in order to better assess the total or overall effects due to adverse weather. A 10-step color code is then assigned based on the magnitude of the impacts. The PWS provides a capability of quantifying the output of the IWEDA rules' degree of impact by computing a composite impact score that, to a certain extent, portrays better resolution within the categories. When used with the Army mission command rules set, the PWS prioritizes and weights the parameters, allowing commanders and intelligence analysts greater flexibility in their mission planning. The PWS will be able to enhance the functionality and maximize the inherent capabilities of the next generation of IWEDA, called the My Weather
Impacts Decision Aid (MyWIDA), currently under development by the ARL.

The PWS has been vetted internally at the ARL and presented to several Army research and development, test and evaluation, and weather community forums, and has received much constructive feedback. Comments received during these presentations have centered on exercising caution in allowing PWS users to randomly change parameter weights without providing some instruction in the context and reasoning for doing so; and there has been some discussion about researching other approaches for computing the CIS. ARL plans to implement the PWS in the MyWIDA version 2.0 web service application in 2014, where it will undergo experimentation and evaluation. Ultimately, the MyWIDA version 2.0 software with the PWS will be incorporated into the Distributed Common Ground Station-Army Weather Services intelligence analyst workstation (the IMETS follow-on system), where it will be used in a battlefield operational setting.

Ongoing work is directed toward extending the PWS concept to account for how much the thresholds are being exceeded by the forecast values in order to infer an impact magnitude. Including this feature will enable the PWS to more fully display high resolution. Also, work is underway to produce separate CIS arrays for "friendly" and "enemy" mission or countermission areas. By differencing these arrays, it will then be possible to assess whether United States and coalition forces will hold the advantage over the enemy on the battlefield in terms of the severity of adverse weather impacts.

Acknowledgements. The authors thank our ARL colleague, Mr. Jeff Johnson, for his technical review of the manuscript and helpful suggestions in preparing it for publication.

\section{REFERENCES}

Department of the Army, 1992: Battlefield weather effects. Field Manual 34-81-1, Washington, D.C., 175 pp. , 2006: Accreditation of the Integrated Weather Effects Decision Aid (IWEDA) software and associated rules database. Memorandum, Department of the Army, Deputy Director, Combat Developments, U.S. Army Intelligence Center and Fort Huachuca, AZ. 5 pp.

Raby, J., R. J. Szymber, and R. Brown, 2003: Development of a methodology for determining the value-added of high temporal and spatial resolution model weather 
forecasts over standard mesoscale model weather forecasts. Army Research Laboratory Technical Report ARL-TR-3116, White Sands Missile Range, NM, 68 pp. [Available from Director, U.S. Army Research Laboratory, ATTN: RDRL-CIE-M (Mr. Jameson), Computational and Information Sciences Directorate, White Sands Missile Range, NM 88002-5501.]

Sauter, D., M. Torres, J. Brandt, and S. McGee, 1999: The Integrated Weather Effects Decision Aid: A common software tool to assist in command and control decision making. Proceedings of the Command and Control Research and Technology Symposium, U.S. Naval War College, Newport, RI, 8 pp. [Available online at dodccrp.org/html4/events_past.html.]

Szymber, R. J., 1997: Owning the weather - An Army force multiplier. The Federal Plan for Meteorological Services and Supporting Research, Fiscal Year 1998, FCM P1-1997, Office of the Federal Coordinator for
Meteorology, Washington, D.C., 4-1-4-7. [Available online at www.ofcm.gov/fedplan/fp-fy98/fedplan.htm.] , 1998: Army weather effects critical values database. Technical Document 2989, Proceedings of the 1997 Battlespace Atmospherics Conference, Space and Naval Warfare Systems Center, San Diego, CA, 259-264.

, 2008: U.S. Army tactical weather and environmental data requirements, critical threshold values, and impacts on operations and systems, and the Integrated Weather Effects Decision Aid (IWEDA) rules. Army Research Laboratory Technical Report ARL-TR-4672, White Sands Missile Range, NM, 179 pp. [Available from Director, U.S. Army Research Laboratory, ATTN: RDRL-CIE-M (Mr. Jameson), Computational and Information Sciences Directorate, White Sands Missile Range, NM 88002-5501.] 